\title{
The Selection Of Wavelet Function In Singular Signal Detection
}

\author{
Chen Zaiyu \\ Faculty of Information Engineering and Automation \\ Kunming University of Science and Technology \\ Kunming, China \\ chenzaiyu-1987@163.com \\ Liu Yang \\ Faculty of Information Engineering and Automation \\ Kunming University of Science and Technology \\ Kunming, China \\ czy1987918@163.com
}

\author{
Liu Zengli \\ Faculty of Information Engineering and Automation \\ Kunming University of Science and Technology \\ Kunming, China \\ liuzengli@hotmail.com \\ Tang Hui \\ Faculty of Information Engineering and Automation \\ Kunming University of Science and Technology \\ Kunming, China \\ thweibo@163.com
}

\begin{abstract}
In the research of singular signal detection, the selection of wavelet function is the first issue to be considered. How to choose the wavelet function for singular signal detection is emphasized in this paper. It's based on the fact that the wavelet function can be perfectly selected by comprehensive considering the characteristics of wavelet function, such as compact support, regularity and vanishing moments. The simulation is presented to illustrate the validity and feasibility of the method.
\end{abstract}

Keywords-singular signal detection; wavelet transform; wavelet function; the characteristics of wavelet function

\section{INTRODUCTION}

The singular points of the singular signal and irregular mutation part often carry an important information which is one of the main characters of a singular signal. As in fault diagnosis, failure usually appears as the mutations of output signal, and thus the determination of the vibration signal singular point is of great significance for the mechanical fault diagnosis [1]. Compared with the traditional analysis method based on Fast Fourier Transform (FFT) which only provides the description of the whole signal singularity, wavelet transform can not only detect the signal singularity size, but also can more accurately determine the position of the singular point. Singularity detection method proposed by Mallat, which is based on wavelet modulus maxima is the most widely used. The method based on singularity theory, utilizing the differences in singularity of signal and noise, by the wavelet transform modulus maxima along the scaling direction of different propagation characteristic can separate noise and reconstruct the original signal. This kind of analysis method in fault diagnosis has achieved fruitful effect.

However, in the singularity detection, the selection of wavelet function is the most difficult thing. In the Wavelet transform, wavelet function only needs to meet certain conditions, even doesn't have a clear expression, so wavelet function has not only irregularity and nonuniqueness. The waveforms of different wavelet functions are very different, and its support length and regularity are also not identical.
Therefore, on the same signal selection of different wavelet function and transform, after processing the results will have big difference. How to select the wavelet function, there hasn't been a unified theory standard yet so far. In practical application, we select wavelet with experience according to the different signal processing purpose [2]. Nevertheless, through the domestic and foreign relevant information, we learn some scholars still have done some exploratory work in this regard. For example, literature [3] selects the wavelet function based on vanishing moments of wavelet: if the detected signal singularity is $\alpha, n-1<\alpha<\mathrm{n}$, you need a above $\mathrm{n}$ vanishing moments of the compactly to support wavelets. Literature [4] presents a wavelet function selection measure based on the similarity of wavelet's regularity coefficient. That is, we can use a wavelet with great regularity coefficient to analyze smooth signal; and use a wavelet with low regularity coefficient to analyze the singular signal. In the paper, several the Db family wavelet functions are chosen, and the wavelet function is perfectly selected through comprehensive consideration the characters of wavelet function, such as compact support, regularity and vanishing moments. The simulation is presented to illustrate the validity and feasibility of the method.

\section{WAVELET TRANSFORM AND DAUBECHIES (DBN) WAVELET}

\section{A. WAVELT TRANSFORM}

The basic idea of wavelet analysis is to represent or approximate a signal or function with a family of function called wavelet function system, which is structured by the translation and telescopic of a basic wavelet function, and of which the conversion coefficient can be used to describe the original signal. The base of wavelet transform is given by

$$
\psi_{\alpha, \beta}(\xi)=|\alpha|^{-1 / 2} \psi((\xi-\beta) / \alpha) \alpha, \beta \in \mathrm{P}, \alpha \neq 0
$$


Where $\psi(\mathrm{x})$ is the basic wavelet function, $\mathrm{a}$ and $\mathrm{b}$ are the scaling and translation factor.

The wavelet transform about the function $\mathrm{f}(\mathrm{t})$ is defined as follows:

$$
W_{f}(a, b)=f * \psi_{a, b}(x)=\frac{1}{\sqrt{a}} \int_{R} f(\mathrm{x}) \bar{\psi}\left(\frac{x-\mathrm{b}}{a}\right) d t
$$

Where $f(\mathrm{t}) \in \mathrm{L}^{2}(\mathrm{R})$ and $*$ denotes convolution. It is observed from (2) that $W_{\mathrm{f}}(a, b)$ have contributions from the scaling factor $a$ and the space position $b$ as a function of wavelet transform. The wavelet transform can be used to analyze signal by the function $\psi(\mathrm{x})$ stretches in scale and translates in spatial domain (time domain).

\section{B. Daubechies (DBN) wavelet}

Db's family wavelet functions are constructed by famous scholars Daubechies, in addition to the Db1 wavelet, other wavelet functions have no explicit expressions. Usually Daubechies wavelet base is denoted as $\mathrm{dbN}, \mathrm{N}$ is serial number, and $\mathrm{N}=1,2, \ldots \mathrm{N}$. The $\mathrm{DbN}$ function is compactly supported standard orthogonal wavelet, it appears makes the discrete wavelet analysis become possible [1].

TABLE I.

Characteristics OF DB WAVELET FUNCTION

\begin{tabular}{|c|c|c|c|c|c|}
\hline \multirow{2}{*}{$\begin{array}{c}\text { Wavelet } \\
\text { Function }\end{array}$} & \multicolumn{5}{|c|}{ Characteristics Of Db Wavelet Function } \\
\cline { 2 - 6 } & Orthogonaliy & $\begin{array}{c}\text { Regularity } \\
\text { coefficient }\end{array}$ & $\begin{array}{c}\text { Supportin } \\
\text { g length }\end{array}$ & $\begin{array}{c}\text { Vanishing } \\
\text { moments }\end{array}$ & Symm \\
\hline $\mathrm{Db} 1$ & Yes & 0 & 1 & 1 & $\mathrm{~N}$ \\
\hline $\mathrm{DbN}$ & $\mathrm{Yes}$ & $\mathrm{N} / 5$ & $2 \mathrm{~N}-1$ & $\mathrm{~N}$ & $\mathrm{~N}$ \\
\hline
\end{tabular}

\section{THE CHARACTERSTICS OF THE WAVELET FUNCTION AND SIGULAR POINT DETECTION}

\section{A. The characterstics of the wavelet function}

In the field of signal processing, the characteristics of the wavelet function are mainly described from the following aspects:

1).Orthogonality: It refers to the components of low frequency signal decomposition (reconstruction) and high frequency decomposition (reconstruction) are orthogonal, when using wavelet function to analyse signal. The strict orthogonality is beneficial to accurate reconstruction of the wavelet decomposition coefficient.

2).Symmetry: Symmetry concerns whether wavelet filter characteristics have linear - phase, which is closely related to the distortion problem. Symmetry of Scaling function and wavelet function can be structured compact branch of the regular wavelet base, which will have linear-phase.

3).Compact Support: Compact support ensures excellent time-frequency local characteristics, and also is conducived to the realization of the algorithm. If the wavelet function $\varphi(t)$ has Compact support, thus the base function is compactly supported. Compactly supported wavelet function is important because it can provide coefficient limited filters in the digital signal analysis process of discrete wavelet decomposes; noncompactly supported wavelet in the actual operation must be cut short. Because the compact supported wavelet can melt the energy of the decomposed signal away, which will results in identification error, theoretically wavelet function with compact support should be chosen in the practical application of singular point recognition. Support length of Db's family wavelet functions are variable, therefore, in the application analysis result will have a certain difference by using different Db's family wavelet functions.

4).Regularity: Regularity is a description of the function's smooth degree, and also a measure of the concentration of the function's energy frequency domain. A function's regularity is given as follows:

$$
|\psi(t+\beta)-\psi(t)|<c|\beta|^{\partial}
$$

Where $0<\mathrm{a}<1$ and $\mathrm{t}, \beta \in R$.c is a constant. Thus, $\mathrm{a}$ is defended as number of regularity, If the $\mathrm{N}$ derivatives of $\psi(t)$ meet the (3), and $\mathrm{r}=\mathrm{N}+\mathrm{a}$, then we can say $\psi(t)$ has regularity ${ }^{[5]}$. The size of the wavelet coefficients reflects a similar degree between the wavelet and function. At the same time the regularity of function and wavelet expresses their differentiability and smooth degree, so according to the similarity, we can use smooth wavelet, which namely has great regularity coefficient, to show smoloth functions; and use non-smooth wavelet, which namely has low regularity coefficient, to show the Nonon-smooth function. Those who need a specification are $\mathrm{No}^{\text {the }}$ similarity is not absolutely equal or very close, but a

5).Vanishing Moments: The physical meaning of vanishing moments can be seen as a convergence rate used the wavelet function approximates a signal.

For any $0 \leq \mathrm{m} \leq \mathrm{p}$ and $\mathrm{m}, \mathrm{p} \in \mathrm{R}$. Thus

$$
\int_{R} t^{m} \varphi(t) d t=0
$$

The wavelet function has $\mathrm{p}$ vanishing moments. Vanishing moments show that the degree of concentration of the energy in the wavelet transform. Theoretically the greater the number is, the stronger the ability of the wavelet transform signal reflects the high frequency detail is.

\section{B. Wavelet singularity detection}

Usually, we can use lipschitz index a to describe the local singularity of the signal. The greater $\mathrm{A}$ is, the more smooth the function is; the smaller a is, the more the function singularity is. The basic principle of detection is when nearby the singular point the signal's lipschitz index a $>0$, the wavelet transform modulus maxima increases with the scale; and a $<0$,it decreases with the increase of the scale. That is to say in an appropriate scale, according to the relationship between wavelet transform modulus 
maxima and singular point, the signal singular point location can be analyzed.

\section{CHOOSING A WAVELET BASE}

The above is the theoretical standard of selecting wavelet function, but in the practical application the concrete problem should be made a concrete analysis. When we choose wavelet base in the process of the singular signal detection, wavelet transform can effectively detect the number and position of fault points, and can eliminate the impact of various interference noise.

Thus in the process of choosing wavelet base, compactly supported wavelet bases are the primary choice. The smaller the support size is, the stronger the wavelet function's ability that reflects the local characteristic is. In order to effectively determine the mutation point, we should choose to wavelet bases which have a small size support; however, the support size can't too small, we need reasonable choice according to the actual situation. Secondly, Wavelet base must have many vanishing moments. The number of vanishing moments is closely related to Lipschitz index. In order to effectively highlight the characteristics of the singularity, a certain number is necessary. However, too high number will increase calculation. In addition, regularity is also a very important rule, because the regularity of function and wavelet can express the respective differentiability and the smooth degree. We can use smooth wavelet, which namely has high regularity coefficient, to show smooth functions; and use non-smooth wavelet, which namely has low regularity coefficient, to show the non-smooth function. But this is not absolute. For symmetry and orthogonality, in the analysis of mutation signal, people are more concerned with singular point position, generally does not require signal reconstruction, so the requirement of both can be widen.

In the singular mutation signal detection, due to its signal features are very irregular, we must comprehensive consider all the characteristics to choose a suitable wavelet function, then it can achieve better recognition results. To sum up, according to the above analysis, we should select the wavelet function by considering the wave's regularity, compact support set and number of vanishing moments. In order to verify the effectiveness of different wavelet bases, we have constructed an experimental signal, shown in Fig.1.

For a given mutation signals, when we select wavelet base, we must consider compact support of time -frequency domain field first. Due to the wavelet db have a compact support of size $2 \mathrm{~N}-1, \mathrm{db} 1, \mathrm{db} 2, \mathrm{db} 3$ and $\mathrm{db} 4$ wavelet base's support sizes are smaller, it is said that they have better local characteristic; secondly, the $\mathrm{dbN}$ wavelet function $\psi$ has $\mathrm{N}$ vanishing moments, the theory shows the greater the number of vanishing moments is, the stronger the wavelet transform's ability that can reflect the signal's high frequency detail is . But in the singular mutation signal detection, according to the requirements of the above analysis, $\mathrm{N}$ don't have too much; In addition, regularity is a also very important rule. In order to effectively analyze the mutation signal, we should choose a wavelet base function that has small regularity .From Tab. 1 we can see the regularity coefficient of $\mathrm{db}$ wavelet increase with $\mathrm{N}$. Therefore, through comprehensive consideration of the above three aspects, db3 wavelet is probably the most suitable wavelet function. In order to verify the above analysis, we use the different $\mathrm{dbN}$ wavelet function to detect the singular original signal and decompose it into 4 layers.

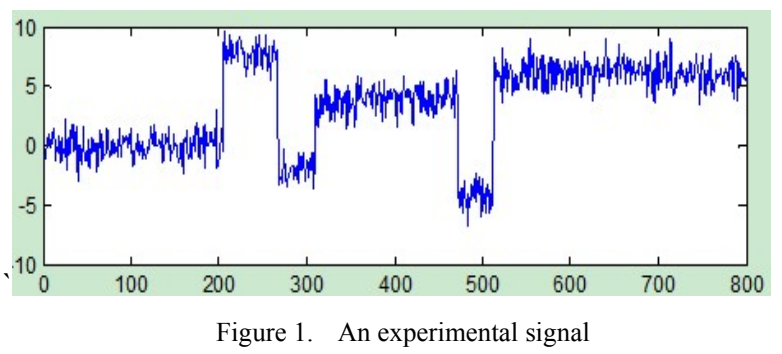

Fig. 1 is the original signal, we can see obviously five mutation points among 0:800: two between 200:30, one between300:400, one between400:500 an'd one between500: 600. Further using the wavelet of $\mathrm{db} 2, \mathrm{db} 3$, $\mathrm{db} 4, \mathrm{db} 5$ decompose the original signal into 4 layer, we can get four layers detail signal and the fourth floor approximate signal, as shown in Fig.2, Fig.3, Fig.4 and Fig.5. By contrast, from the Fig. 3, we clearly see five mutation points in the second layer details signal which is decomposed by the $\mathrm{db} 3$ wavelet, and position is correspond with the mutation points of original signal. And db3 wavelet which has smaller compact support, regularity and larger number of vanishing moments, is consistent with the above analysis. So the method of elect the wavelet base based on comprehensive considering the wavelet function's characteristics, such as compact support, regularity and order of vanishing moments is feasible.

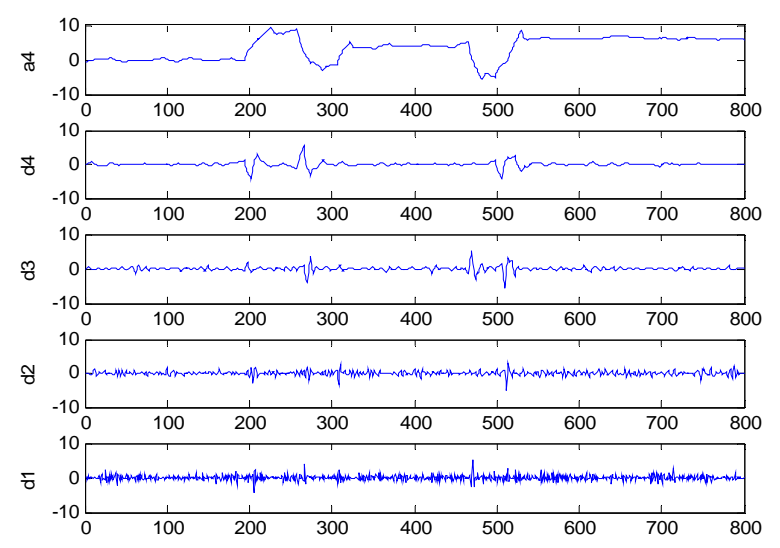

Figure .2. Signal decomposition with $\mathrm{db} 2$ wavelet 


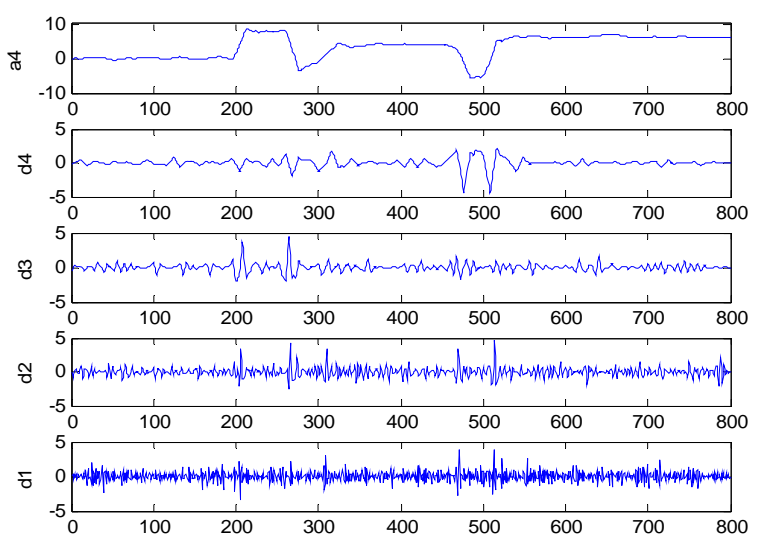

Figure.3. Signal decomposition with $\mathrm{db} 3$ wavelet

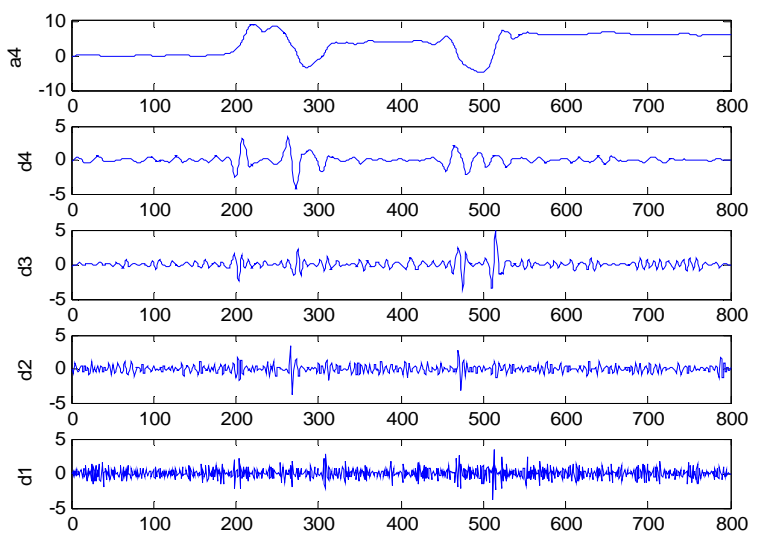

Figure.4. Signal decomposition with db4 wavelet

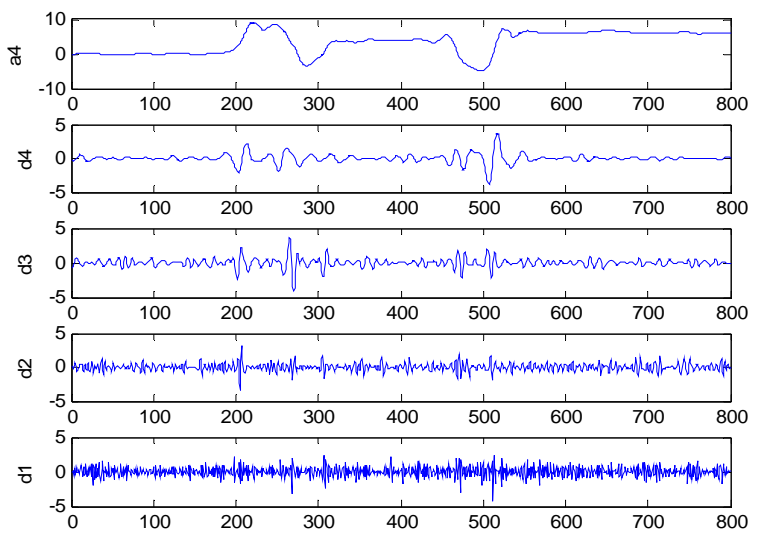

Figure.5. Signal decomposition with db5 wavelet

\section{CONCLUSION}

Singular Signal Detection based on wavelet transform modulus maxima can be identified the number and position of fault point. The simulation shows in singular signal detection, considering the characteristics of wavelet function to select the wavelet is feasible, no matter abrupt signal or slowly varying signal detection. In the influence factors of wavelet, such as orthogonality, compact support, regularity, order of vanishing moments, symmetry, orthogonality and symmetry is not dominant; but regularity, compact support and order of vanishing moments are dominant. Therefore, in the detection, we should accord to the signal characteristics, and comprehensively consider the wavelet function's regularity, compact support and order of vanishing moments, to select the appropriate wavelet. Although this method is only combined with Daubechies wavelet to explain, other department of wavelet is adaptive also.

\section{ACKNOWLEDGMENT}

I am very sincerely grateful to my tutor for his careful instruction and support. Moreover, I also thank all partners in the laboratory for their enthusiastic help and advice.

This work was supported by Natural Science Foundation Project of NSFC under the grant No.60872157 and No.61271007

\section{REFERENCES}

[1] Zhang Defeng.MATLAB and Wavelet Transform [M].Bei jing:CHINA MACHINE PRESS. 2012,PP 263-275

[2] Chen Xiping,Mao Haijie, Li Wei. Study on Choosing Mother Wavelet for Signal Singularity Detection Basedon MATLAB[J]. Computer simulation, Vol 21.Nov.2011,pp 251-254.

[3] Zhang Zhiyong.MATLAB Tutorial[M].Bei jing:BEI JING UNIVERSITY PRESS.2002.pp 125-130.

[4] Zhou Xiaoyong,Ye Yinzhong.Selection of Wavelet Base in Fault Diagnosis[J].Control engineering of China,Vol 10,No.4.Jul.2003, pp134 -139

[5] Yang Xiaonan, Tang Hesheng, Chen Rong, Xue Songtao. Selection on Db'S Family Wavelet Functionsin Steel Structural Damage Identification[J].JOURNALOF TONGJI UNIVERSITY (NATURAL SCIENCE)Vol.34No.12. Dec.2006,pp89-95 